\title{
Instrumentation et gestion numérique des temps morts pour la métrologie de la radioactivité
}

\section{Digital instrumentation and dead-time processing for radionuclide metrology}

\author{
Benjamin CENSIER, Christophe BOBIN et Jacques BOUCHARD
}

CEA, LIST, Laboratoire national Henri Becquerel (LNE-LNHB), 91191 Gif-sur-Yvette, France, christophe.bobin@cea.fr.

\section{Résumé}

La plupart des chaînes d'acquisition utilisées en métrologie de la radioactivité font appel à des modules électroniques au standard NIM. Ce type d'instrumentation analogique bénéficie de plusieurs décennies de pratique et de savoir-faire, et constitue donc une référence dans le domaine. Cependant, afin d'une part d'assurer la pérennité des installations, d'autre part de bénéficier de nouvelles fonctionnalités, le développement de nouvelles chaînes d'acquisition s'appuyant sur la numérisation et le traitement numérique semble aujourd'hui incontournable. Cet article présente tout d'abord les différentes technologies numériques utilisables pour l'instrumentation. Dans la deuxième partie, un état de l'art du domaine est présenté en insistant sur le rôle fondamental du traitement des temps morts. La dernière partie est consacrée à la présentation de deux systèmes développés au LNE-LNHB, le premier équipant un dispositif cristal-puits $\mathrm{NaI}(\mathrm{Tl})$, le second un ensemble de trois photomultiplicateurs en vue de l'application des méthodes RCTD et des coïncidences $4 \pi \beta-\gamma$.

MOTS CLÉS : MÉTROLOGIE DE LA RADIOACTIVITÉ, INSTRUMENTATION NUMÉRIQUE, TEMPS MORT, CRISTAL-PUITS, COÏNCIDENCES, MÉTHODE RCTD.

\footnotetext{
Abstract

Most of the acquisition chains used in radionuclide metrology are based on NIM modules. These analogue setups have been thoroughly tested for decades now, becoming a reference in the field. Nevertheless, the renewal of ageing modules and the need for extra features both call for the development of new acquisition schemes based on digital processing. In this article, several technologies usable for instrumentation are first presented. A review of past and present projects is made in the second part, highlighting the fundamental role of dead-time management. The last part is dedicated to the description of two digital systems developed at $L N E-L N H B$. The first one has been designed for the instrumentation of a $\mathrm{NaI}(\mathrm{Tl})$ well-type crystal set-up, while the second one is
}

used for the management of three photomultipliers in the framework of the TDCR method and as a part of the development of a digital platform for coincidence counting.

KEY WORDS: RADIONUCLIDE METROLOGY, DIGITAL INSTRUMENTATION, DEAD-TIME, WELL-TYPE CRYSTAL, COINCIDENCE COUNTING, TDCR METHOD.

\section{Introduction}

En métrologie de la radioactivité, l'instrumentation analogique utilisée dans la plupart des chaînes de mesure est progressivement confrontée à des problèmes de disponibilité et de prix. Dans le même temps, le domaine de l'électronique numérique et plus précisément la numérisation rapide des signaux ainsi que le traitement numérique du flot de données généré ont connu des progrès importants, autorisant l'utilisation de ces technologies pour le développement de chaînes d'acquisition. Outre le problème posé de la pérennité des installations, ces technologies permettent d'envisager l'ajout de fonctionnalités par rapport aux installations existantes.

La figure 1 illustre le principe général de conception d'une chaîne d'acquisition dite "numérique », par opposition aux chaînes analogiques classiques. L'idée générale consiste à numériser le signal au plus près du détecteur, puis de faire traiter le signal numérisé en temps réel par des composants programmables afin d'en tirer les informations d'intérêt. Ce schéma type autorise de nombreuses variantes, suivant la position de la numérisation 


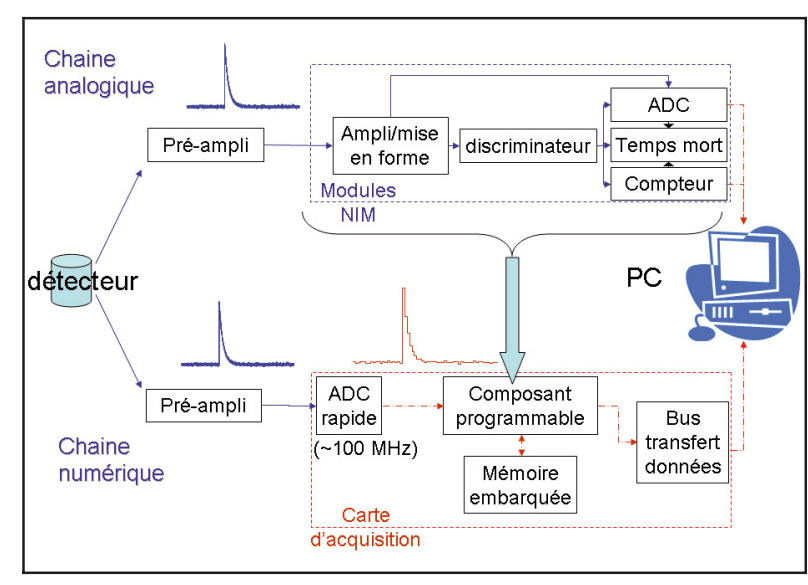

Fig. 1. - Schéma type d'une chaîne d'acquisition analogique et d'une chaîne numérique équivalente où toutes les fonctions réalisées par les modules analogiques sont implémentées dans un composant programmable. Les flèches en trait continu indiquent la transmission d'un signal analogique, les flèches en pointillés indiquent la transmission d'un signal numérique.

dans la chaîne d'acquisition, ou encore la répartition des fonctions d'analyse entre les parties « en ligne» (composants programmables) et «hors ligne» (ordinateur d'acquisition). Ces différentes possibilités, ainsi qu' une brève présentation des technologies de numérisation et de traitement en temps réel sont discutées dans la section 2.

L'implémentation de fonctions sur composants programmables ouvre de nombreuses possibilités, qui sont parfois soit impossibles soit compliquées et coûteuses à mettre en œuvre sur une chaîne analogique. En pratique, il est cependant nécessaire de fonder cette implémentation sur le savoir-faire acquis depuis maintenant quelques décennies avec l'instrumentation analogique. D'une part, un système numérique nécessite toujours un dispositif électronique analogique d'entrée pour adapter les signaux de sortie du détecteur. D'autre part, on insistera particulièrement dans cet article sur la notion de temps mort de mesure et sur sa gestion, élément central et primordial pour une mesure fiable de l'activité d'un radionucléide. Cette notion est introduite dans la section 3. La section 4 présente un état de l'art de l'instrumentation numérique appliquée à la métrologie de la radioactivité. On y décrira plus particulièrement deux plates-formes numériques développées au LNE-LNHB. La première concerne un dispositif de mesure $4 \pi-\gamma$ doté d'un cristal-puits $\mathrm{NaI}(\mathrm{Tl})$ et réalise la numérisation du signal et le traitement en ligne du déclenchement ainsi que des mesures d'amplitude et de temps. L'analyse des données est effectuée par un ordinateur personnel (PC) d'acquisition, notamment le traitement des temps morts. La deuxième est conçue pour traiter les signaux de discrimination en provenance d'un compteur à scintillation liquide à trois photomultiplicateurs dans le cadre de l'application de la méthode RCTD (rapport des coïncidences triples à doubles). Dans ce cas, la logique de comptage des coïncidences entre les différentes voies est assurée en temps réel par un composant programmable. L'ajout d'une voie gamma à cette deuxième plate-forme en combinaison avec le compteur à scintillation liquide, permettra à terme de disposer d'un système de mesures en coïncidences $4 \pi \beta-\gamma$.

\section{Technologies de numérisation et de traitement numérique}

\subsection{Numérisation}

Les caractéristiques techniques des numériseurs (Analog-to-Digital Converter ou ADC) ont connu une progression importante à la fois en termes de profondeur de codage (nombre de bits sur lequel la numérisation est effectuée) et de fréquence d'échantillonnage. Les numériseurs commerciaux les plus performants ont aujourd'hui des fréquences qui atteignent $1 \mathrm{GHz}$ avec plus de 10 bits (soit 1024 niveaux). Chaque type de numériseur offre un compromis particulier entre la vitesse d'échantillonnage et la profondeur de codage.

La contrainte sur la fréquence d'échantillonnage peut être fixée par la bande passante du signal à numériser (critère de Nyquist-Shannon) et par la précision désirée sur les mesures de temps. Il est en effet important de noter que l'occurrence des évènements à détecter est asynchrone comparée à l'horloge utilisée pour l'échantillonnage : le début d'une impulsion ne coïncide pas en général avec un front de cette horloge. Si le déclenchement est effectué sur le signal numérisé, en repérant les échantillons dépassant un seuil donné, il y a donc un écart variable entre le temps d'occurrence déterminé sur le signal numérisé et le « vrai » temps d'occurrence du signal analogique. Cet écart est bien sûr d'autant plus petit que la fréquence d'échantillonnage est grande. D'autre part, si les mesures de temps nécessaires au traitement des temps morts (intervalles de temps entre évènements et durées de discrimination) sont aussi effectuées sur le signal numérisé, la fréquence d'échantillonnage jouera également un rôle dans l'exactitude de la mesure du temps actif associé à une période de comptage. On peut cependant obtenir une mesure totale de temps actif avec une exactitude inférieure à la période d'échantillonnage, en raison du caractère aléatoire des occurrences des désintégrations radioactives.

La profondeur de codage nécessaire peut être fixée par le rapport signal sur bruit désiré (directement lié à la résolution en énergie en spectrométrie) et par la dynamique d'entrée souhaitée. Une contrainte importante pour la spectrométrie est la linéarité du numériseur. Cette linéarité, ainsi que les différents bruits intervenant dans la conversion, limitent la profondeur « effective » de codage. On peut définir un nombre de bits effectif (ENOB : Equivalent Number of Bits), par définition inférieur au nombre de bits théorique, en prenant en compte ces limitations. En pratique, la plupart des mesures à haute résolution en énergie (diodes germanium, bolomètres..) utilisent une numérisation sur 14 à 16 bits. D'autres mesures faisant intervenir de plus faibles résolutions peuvent se limiter entre 10 et 12 bits (cristal scintillateur NaI(Tl)). Enfin certaines applications comme la scintillation liquide, produisant des signaux de basse résolution en énergie 
impliquant des cadences de mesure rapides (de l'ordre de quelques nanosecondes), peuvent soit utiliser une discrimination analogique produisant des signaux au format logique directement utilisables par un système électronique numérique, soit tirer parti des numériseurs les plus rapides ( $\sim 8$ bits et quelques gigahertz).

\subsection{Traitement numérique en ligne et hors ligne}

Si l'on prend l'exemple typique d'un numériseur à $100 \mathrm{MHz}$ sur 12 bits, on obtient un flux de données en sortie du numériseur de l'ordre de 150 millions d'octets par seconde. Un tel flux réclame une étape de traitement capable de l'analyser sans ajout de temps mort. On trouve de telles capacités dans le domaine des composants programmables, éléments dédiés au calcul en temps réel sur de grands débits de données. On distinguera ici deux grandes familles de composants programmables :

- Les DSP (Digital Signal Processor) sont des processeurs dédiés au calcul en temps réel. Leur architecture est proche des processeurs équipant les ordinateurs, mais bénéficie de fonctions et de bibliothèques dédiées. On en trouve des exemples sur les cartes graphiques récentes disposant de possibilités de calcul indépendantes du processeur central. Leur programmation est proche de la programmation usuelle (utilisation du langage C) mais nécessite tout de même des environnements de programmation dédiés (ex : Code Composer Studio) et une bonne connaissance des fonctions matérielles et logicielles spécifiques au composant utilisé.

- Les FPGA (Field Programmable Gates Array) sont des matrices de blocs logiques interconnectés, les connexions entre les blocs pouvant être activées ou inhibées par programmation afin de réaliser la fonction désirée. L'implémentation est effectuée dans un langage spécifique (VHDL ou Verilog) permettant la description d'un circuit électronique numérique. La programmation effective du FPGA nécessite un environnement de programmation capable d'effectuer les étapes de simulation (simulation d'un circuit équivalent pour valider les fonctions développées) et de synthèse (définition de la carte des connexions à inhiber sur le composant pour réaliser la fonction décrite par le code).

Dans les deux cas, un développement pour le traitement en temps réel implique une expertise à la fois logicielle et matérielle, l'optimisation d'une fonction faisant aussi bien appel à l'algorithmie qu'à la connaissance des fonctions physiquement implantées dans le composant ${ }^{1}$. Pour schématiser les différences essentielles entre ces deux types de composants, on peut dire que les DSP sont plus

1 Mise à part l'étape d'implémentation proprement dite, l'étape d'intégration d'un composant programmable au sein d'un système (dialogue avec une mémoire embarquée et avec le PC d'acquisition, gestion des différents protocoles...) peut s'avérer particulièrement délicate pour un novice ou pour un professionnel ne connaissant pas le système en question. proches de la programmation habituelle et en ce sens plus simples à utiliser. La programmation d'un FPGA nécessite en effet des connaissances aussi proches de l'électronique numérique que de la programmation proprement dite. En revanche, le fonctionnement d'un DSP est par nature séquentiel (mise à part la possibilité croissante de disposer de plusieurs cœurs en parallèle), là où un FPGA est naturellement parallèle car étant conçu pour émuler les fonctions d'un circuit électronique numérique. Il est donc courant chez les développeurs d'implémenter et de tester une fonction sur un DSP avant de la faire migrer et de l'adapter à un FPGA pour gagner en vitesse de traitement effective. On assiste en outre à une convergence entre les deux technologies, les dernières générations de FPGA intégrant physiquement un ou plusieurs cœurs de processeurs dans leur structure.

Parmi les choix de conception d'une instrumentation numérique, on doit définir les fonctions à réaliser en temps réel par un composant programmable et les fonctions éventuellement implémentées hors ligne sur un PC d'acquisition. En extrayant l'information utile du flot de données initial, le composant programmable réalise une compression de données sans perte lors du transfert vers le PC d'acquisition. Cette réduction est en effet utile voire incontournable dans la plupart des cas. D'une part, les bus de transfert de données ont des bandes passantes limitées et des débits effectifs pouvant fluctuer au cours du temps, d'autre part le stockage en mémoire volatile ou permanente de grandes quantités de données peut vite s'avérer problématique. Le traitement hors ligne a donc l'avantage de ramener l'implémentation sur le terrain connu de l'ordinateur personnel et d'autoriser le stockage de données pour traitement ultérieur, mais la conception du système doit prévoir, d'une part un pré-traitement en temps réel réduisant le flux de données, d'autre part un lien efficace entre l'acquisition et le PC (liens DMA (Direct Memory Access), utilisation d'un fichier tampon...). Ces deux points découlent de la problématique centrale en métrologie de la radioactivité : la maîtrise des temps morts de mesure.

\subsection{Cartes commerciales}

Plusieurs solutions commerciales existent et satisfont, en première approche, la plupart des contraintes de la métrologie de la radioactivité en termes de numérisation et de puissance de calcul. Cependant, deux points cruciaux sont souvent inadaptés : le dispositif électronique d'entrée et le traitement des temps morts. Les systèmes commerciaux développés spécifiquement pour les applications de comptage et de spectrométrie présentent de nombreuses fonctionnalités intéressantes, et sont directement adaptés à la plupart des détecteurs concernant le dispositif électronique d'entrée, mais le traitement des temps morts est la plupart du temps mal documenté (méthodes de corrections utilisées, différentes sources de temps mort du système...) et/ou mal adapté à une mesure de précision métrologique.

D'autre part, il existe de nombreuses solutions commerciales de cartes d'acquisition programmables, 
réunissant les fonctions de numérisation et de traitement. Ces solutions offrent un système déjà intégré sur le plan numérique, et permettent d'implémenter un traitement de temps mort adapté. Cependant, elles sont la plupart du temps développées pour des applications de télécommunications à hautes fréquences. Leur dispositif électronique d'entrée est de ce fait souvent inadapté à la bande de fréquences et à la dynamique d'entrée nécessaire (basses fréquences atténuées en entrée, faible dynamique de l'ordre de $1 \mathrm{~V}$ ou inférieure...).

\section{Traitement des temps morts}

\subsection{Définition}

La mesure d'un taux de comptage consiste à diviser un nombre d'évènements détectés pendant un certain laps de temps par le temps de mesure. Il convient de préciser cette notion de temps de mesure : il s'agit du temps pendant lequel le système est considéré apte à détecter un évènement, on l'appelle «temps actif ». En effet, toute instrumentation peut introduire des périodes de paralysie pendant lesquelles le système est considéré comme «aveugle», soit en raison des limitations de la méthode de détection elle-même, soit par les temps d'analyse requis par certaines étapes de traitement [1]. La première source de ces «temps morts » est bien illustrée dans le cadre de la détection sur dépassement de seuil : une fois que le signal a dépassé le seuil de détection, un évènement est détecté, mais toute nouvelle détection est impossible avant le retour du signal en-deçà du seuil. Le temps mort associé dépend de divers paramètres, des caractéristiques du détecteur jusqu'aux conditions de mise en forme du signal. La deuxième source est par exemple rencontrée avec l'utilisation des convertisseurs analogique-numérique inclus dans les chaînes analogiques pour mesurer l'amplitude des évènements détectés (il s'agit ici de convertisseurs beaucoup plus lents que ceux discutés dans la section 2.1, qui réalisent une fonction d'échantillonnage-blocage au sommet des impulsions et non une numérisation du signal en continu). Cette mesure réclame un certain temps d'analyse pour chaque évènement, pendant lequel aucun autre traitement ne peut être effectué, c'est donc un «temps mort ».

La maîtrise de ces périodes de temps mort est un enjeu majeur pour l'exactitude de la mesure d'activité, l'essentiel étant de les identifier de manière suffisamment fine. On distinguera deux attitudes générales face au problème : une première consiste dans une modélisation statistique du temps mort total, la seconde est fondée sur la mesure du temps réel d'acquisition hors temps mort ou temps actif.

\subsection{Méthodes pour l'instrumentation analogique}

Comme évoqué précédemment, le temps mort est appliqué suite à un comptage dans le but de prendre en compte le plus efficacement possible les différentes sources de paralysie dans une chaîne de mesure; en outre, il doit être adaptable aux différents systèmes de détection rencontrés en métrologie de la radioactivité.

Dans une instrumentation classique, le temps mort imposé est de type non reconductible ou fixe. Dans ce cas, le signal de temps mort inhibe toute nouvelle détection pour une période durant laquelle tout comptage est impossible ainsi que la génération d'un nouveau temps mort. Cette instrumentation est au prix d'une complexité des corrections statistiques à apporter, autrement dit de l'estimation statistique du temps mort total lors d'une mesure d'activité. L'utilisation d'un temps mort fixe ${ }^{2}$ nécessite une modélisation statistique qui a été affinée au fil du temps [2]. Selon les systèmes de détection utilisés, la complexité des formules employées augmente rapidement - en particulier dans le cas de la méthode des coïncidences $4 \pi \beta-\gamma-$, mais elle ne peut prétendre à la prise en compte de tous les effets expérimentaux possibles. On citera pour exemple les saturations du système électronique après un dépôt d'énergie important dans le détecteur, qui peuvent être à l'origine de longues périodes de temps mort.

Une alternative mieux adaptée à la diversité des signaux délivrés par les détecteurs correspond à l'utilisation de temps morts reconductibles. Ici le temps mort imposé est déclenché après chaque détection qu'elle se situe pendant une période de temps mort ou non. L'intérêt de cette technique réside dans sa combinaison avec la mesure des périodes de temps mort. On utilise alors une méthode de «temps mort reconductible avec mesure du temps actif ». Comparée à une modélisation statistique, l'expérience montre que cette technique offre une grande robustesse dans sa capacité de protection au regard de la diversité des situations de paralysie d'une chaîne de comptage. En général, on préfèrera étendre le temps mort et connaître précisément sa durée, plutôt que de chercher à le réduire sans le maîtriser totalement.

La mesure de temps actif demande un montage électronique dédié, capable de générer un signal logique à l'état haut pendant les périodes de temps mort [3]. Ce signal peut être vu comme la combinaison par un OU logique des signaux de discrimination (à l'état haut lorsque le signal est au-dessus du seuil de détection) et de temps mort imposé (à l'état haut pendant un temps $t_{\mathrm{m}}$ correspondant à la période de temps mort imposée après chaque déclenchement). La mesure du temps actif se ramène alors au comptage des impulsions d'une horloge, inhibé pendant les périodes de temps mort. Le taux de comptage corrigé du temps mort est alors $N / T_{\mathrm{a}}$, où $N$ est le nombre d'évènements détectés hors temps mort, et $T_{\mathrm{a}}$ est le temps actif total de la mesure. Cette méthode a été mise en œuvre avec succès au LNE-LNHB sous forme de modules électroniques au standard NIM [3] et testée sur plusieurs méthodes de mesure d'activité (cristal-puits, coïncidences $4 \pi \beta-\gamma$, RCTD...).

\footnotetext{
2 La notion de temps mort fixe est critiquable : si le temps mort imposé est fixe, le temps mort réel ne l'est pas, en général.
} 


\subsection{Adaptation à l'instrumentation numérique}

La première contrainte pouvant être fixée à une instrumentation numérique est de ne pas ajouter de temps mort supplémentaire par rapport à une instrumentation analogique équivalente. En d'autres termes, le cheminement du signal numérisé à travers les diverses étapes (numérisation, traitement des données, transfert des données à un PC) doit être sans perte de données. C'est par définition le cas sur un système qui fonctionne uniquement en temps réel. Cependant, un soin particulier doit être apporté aux liaisons permettant le transfert de données avec un PC d'acquisition, dont le débit est le plus souvent variable. On peut intervenir en amont sur la qualité de la liaison (ex : utilisation de canaux DMA pour un accès au PC indépendant des ressources utilisées sur celui-ci), ou en aval, le plus souvent par l'utilisation de mémoires tampon permettant d'assurer la continuité du flux de données en stockant temporairement certaines données, ce qui autorise un point d'accumulation à court terme. Etant donné les possibilités techniques du matériel existant, un système numérique bien conçu peut largement respecter cette contrainte. On obtient alors ce qui est parfois abusivement qualifié de «dead-time free digital chain » : cette expression concerne uniquement le traitement et le transfert des données numériques, l'ensemble de la chaîne de mesure étant toujours astreint au minimum au temps mort naturel du système (correspondant au temps de discrimination).

Une fois cette précaution assurée, l'implémentation de la gestion des temps morts est, à plusieurs points de vue, réalisée plus simplement sur un système numérique. En effet, le traitement sur un signal numérisé donne directement accès à des mesures de temps (temps de discrimination, intervalles de temps entre évènements, datation des évènements). Le comptage des échantillons d'un signal numérisé par une horloge suffisamment stable et précise peut en effet constituer une mesure de temps adéquate. L'utilisation d'une horloge externe étalonnée est également envisageable, mais les horloges utilisées pour la numérisation rapide ont généralement des caractéristiques déjà très intéressantes. Le comptage des détections hors temps mort revient à une simple sélection des évènements séparés de plus de $t_{m}$ de l'évènement précédent ( $t_{m}$ étant la durée du temps mort imposé, qui est alors naturellement reconductible). Ces deux opérations peuvent s'effectuer totalement en temps réel, ou hors ligne sur un PC d'acquisition à partir d'un enregistrement des mesures de temps.

\section{4. État de l'art}

\subsection{Premiers systèmes (de 1996 à 2004)}

Les premiers développements d'instrumentation numérique pour la métrologie de la radioactivité ont concerné la méthode des coïncidences $4 \pi \beta-\gamma$ [4]. Dans cette méthode, on doit détecter les évènements en coïncidence entre deux voies de mesure, l'une sensible au rayonnement bêta (électrons) et l'autre au rayonnement gamma (photons).

La première expérience fut l'objet d'une collaboration entre l'ANSTO (laboratoire national australien) et le NPL (Institut national de métrologie du Royaume-Uni) [5], pour un projet fondé sur une carte d'acquisition commerciale, permettant la numérisation et le stockage sur une mémoire embarquée du signal numérisé. Ce premier système est limité par la profondeur de cette mémoire embarquée, ne disposant d'aucune étape de calcul temps réel pour réduire le flux de données et permettre un transfert continu vers un PC d'acquisition pour traitement. Le KRISS (laboratoire national coréen) a développé dans la même période un système similaire [6], utilisant un contrôleur externe de coïncidences contrôlant le numériseur.

Par la suite, l'utilisation des fonctionnalités temps réel des FPGA a permis une amélioration des systèmes initiaux. La collaboration ANSTO/NPL a notamment permis le développement d'une plate-forme de «Digital Coincidence Counting » (DCC) comprenant deux voies numérisées à $20 \mathrm{MHz}$ sur 12 bits, et un FPGA chargé des fonctions de discrimination et de datation des événements [7]. Le FPGA permet d'envoyer au PC d'acquisition les seules portions de signal qui sont au-dessus du seuil fixé par l'utilisateur, réduisant ainsi le flux de données à envoyer. La forme des impulsions stockées sur disque dur est ensuite traitée sur le PC, qui gère les coïncidences via la datation des évènements par un mot binaire de 40 bits et la mesure d'amplitude des évènements. Etant donné la fréquence d'échantillonnage limitée, le caractère asynchrone des évènements détectés par rapport à l'horloge utilisée peut introduire une dispersion supplémentaire dans les mesures d'amplitude. Cet effet, ainsi que les non-linéarités résiduelles du numériseur, sont corrigés durant l'analyse hors ligne.

D'autres plates-formes développées au CMI (laboratoire national tchèque) [8] et au VNIIFTRI (laboratoire national russe) [9] n'utilisent pas de composants programmables mais une combinaison de modules traditionnels (discrimination, gestion des temps morts, ADC « lent» pour la mesure d'amplitude...) et de techniques numériques (transfert des données sur le PC via DMA, analyses hors ligne).

On notera que tous ces systèmes utilisent la méthode du «temps mort fixe» avec correction statistique du temps mort dont la précision s'avère critique selon les taux de comptage (typiquement au-delà de $10 \mathrm{kBq}$ ). Par ailleurs, cette correction est dans certains cas grandement compliquée par les délais introduits par les étapes de transfert de données [10] : soit on ne dispose pas de composant programmable et les interruptions nécessaires au dialogue avec le PC sont critiques, soit on utilise un composant type FPGA qui réduit le flux de données mais demande toujours le rapatriement de l'ensemble de la forme des évènements au PC d'acquisition. Ces limitations impliquent automatiquement une limite sur le taux 
de comptage maximal atteignable sans perte significative de comptage (autour de 10000 coups par seconde).

\subsection{Développements récents (à partir de 2005)}

Les systèmes plus récents ont bénéficié de l'évolution rapide des technologies de numérisation et de traitement en temps réel. En termes de numérisation, des fréquences d'échantillonnages supérieures à $100 \mathrm{MHz}$ pour des profondeurs de codage entre 12 et 14 bits sont couramment utilisées. En termes de traitement temps réel, l'utilisation de FPGA se généralise, mais la complexité inhérente à leur programmation appelle soit l'utilisation d'environnements de programmation simplifiés (ex : Simulink ou Labview pour la programmation par schémas-blocs et la synthèse automatique de code), soit le recours à une sous-traitance des fonctions en temps réel. Ces deux approches ont chacune leurs avantages et inconvénients respectifs. La programmation par schémas-blocs permet une implémentation en interne mais peut s'avérer limitée pour certaines fonctions demandant une optimisation particulière et une réelle expertise de la programmation. L'appel à la sous-traitance en revanche, introduit une dépendance à une société extérieure mais peut déboucher, sur la base d'un cahier des charges bien conçu, sur un système «sur mesure » et optimisé. Le développement du marché des technologies numériques offre de nombreuses possibilités : adaptation de cartes programmables commerciales et/ou sous-traitance. Cependant, son évolution rapide rend nécessaire une réflexion sur la pérennité des systèmes numériques développés (technologies matérielles et logicielles, utilisation d'algorithmes propriétaires, pérennité des entreprises sous-traitantes...).

\subsection{Exemple d'application : instrumentation d'un détecteur cristal-puits $\mathrm{NaI}(\mathrm{Tl})$}

L'instrumentation numérique développée pour la mesure avec le cristal-puits NaI(Tl) au LNE-LNHB depuis 2006 [11] est un exemple de compromis entre l'appel à la sous-traitance pour les fonctions temps réel, et le développement en interne de fonctions hors ligne. Ce dispositif de mesure a été choisi comme première étape dans le projet de développement de l'instrumentation numérique car ses contraintes en termes de résolution en énergie (supérieure à $10 \%$ ), de nombre de voies (une seule voie de mesure) et de dynamique temporelle des signaux (de l'ordre de la microseconde) sont relativement faibles. Cette instrumentation est fondée sur un système commercialisé par la société Sundance. Il est constitué d'une carte dite «porteuse » au format PCI, équipée de deux modules pouvant être utilisés indépendamment et de manière autonome (stand-alone). L'un des modules a des fonctionnalités purement calculatoires, il est équipé d'un FPGA et d'un DSP. Le second module constitue le cœur du système. Il est équipé de deux numériseurs (200 MHz/12 bits) et d'un FPGA Xilinx Virtex II pro. Son montage électronique d'entrée a été modifié à notre demande de manière à avoir un couplage DC, inexistant pour la plupart des applications comme les télécommunications qui traitent des signaux de hautes fréquences.
Il est remarquable de constater que l'ensemble des fonctions implantées (déclenchement, filtrage, mesures d'amplitude et de temps) occupent moins de $10 \%$ de la capacité totale du circuit FPGA. Le DSP est uniquement utilisé durant la phase de développement, il gère le transfert de données entre le PC hôte et la carte d'acquisition via le bus PCI. Le FPGA est chargé des fonctions temps réel déjà citées : il détecte les dépassements de seuil et détermine pour chaque évènement les informations d'amplitude et de temps. Ces informations constituent le minimum nécessaire pour une mesure d'activité au-dessus d'un seuil en énergie donné, en utilisant la méthode des temps morts reconductibles avec mesure du temps actif. On est alors ramené à des débits de données beaucoup plus faibles, pouvant être transférées sans perte au PC via une zone tampon dans la mémoire vive et/ou dans un fichier de stockage sur disque dur. Cette partie en temps réel a été sous-traitée à la société Sundance. Les fonctions hors ligne, développées au LNE-LNHB, sont la formation des différents histogrammes (spectre en énergie, histogrammes des intervalles de temps actif entre évènements) et la gestion des temps morts qui constitue le cœur de la mesure d'activité. Cette gestion des temps morts est constituée d'un algorithme développé en langage C, Matlab et Labview, suivant l'utilisation désirée.

Les premiers tests concernant la numérisation ont montré la présence d'une non-linéarité en fonction de l'énergie. Ce problème, trouvant son origine dans une mauvaise conception du dispositif électronique d'entrée, illustre bien le genre de limites que peut atteindre la soustraitance : une société spécialisée en électronique numérique n'est pas toujours apte à répondre aux contraintes spécifiques de la métrologie de la radioactivité sur le système analogique (bande passante, linéarité, dynamique d'entrée...). Mais ce problème illustre aussi les possibilités offertes par l'électronique numérique : c'est au prix d'une modification mineure du code implanté dans le FPGA que ces non-linéarités ont pu être corrigées en temps réel sur le signal numérisé. Cette correction entraîne une légère perte de bits effectifs, qui n'est pas rédhibitoire étant données les caractéristiques de résolution en énergie du cristal-puits. À terme, l'élaboration d'un dispositif électronique d'entrée mieux adapté est cependant prévu. Il devra faire le lien entre une sortie de détecteur dont les signaux peuvent atteindre ponctuellement plusieurs volts (cas de l'interaction de rayons cosmiques dans le cristal) et une entrée de numériseur dont la dynamique est de l'ordre de $1 \mathrm{~V}$; d'où la nécessité d'un système d'écrêtage pour protéger le numériseur. Le prototype développé conserve le préamplificateur et l'amplificateur utilisés dans la chaîne analogique (l'amplificateur applique une mise en forme gaussienne aux signaux du préamplificateur à partir de laquelle on mesure une amplitude). L'écrêtage est effectué par deux diodes placées avant entrée de la carte d'acquisition.

En termes de spectrométrie, on observe de légères différences entre les chaînes analogique et numérique. Elles concernent la région voisine du seuil d'énergie et la distribution des amplitudes. Sur la figure 2, on remarque que le pic à $662 \mathrm{keV} \mathrm{du}{ }^{137} \mathrm{Cs}$ (correspondant au rayonnement 


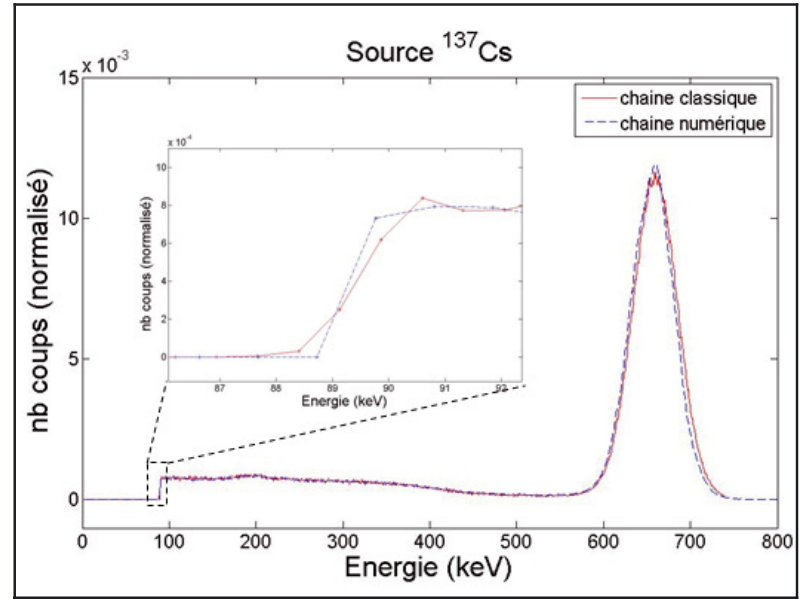

Fig. 2. - Spectre en énergie d'une source de ${ }^{137} \mathrm{Cs}$ sur les chaînes analogique (trait plein) et numérique (pointillés) équipant le dispositif cristal-puits du LNE-LNHB. L'insert montre un agrandissement de la région autour du seuil.

gamma) présente une légère différence dans la distribution des amplitudes mesurées avec la chaîne numérique, et que la forme du spectre près du seuil diffère entre les deux chaînes. Cela est dû au fait que, contrairement à la chaîne analogique, la mesure d'amplitude effectuée sur le FPGA comprend une correction de la ligne de base, consistant à retrancher au maximum de chaque impulsion la valeur moyenne de quelques échantillons précédant le déclenchement. Cette correction permet de mieux mesurer les amplitudes dans certains cas d'empilements d'impulsions ou de variations de la ligne de base.

En termes de comptage, on distinguera deux types de mesure : le premier où les deux chaînes partagent le même signal de déclenchement délivré par un module analogique, et le second où le déclenchement est indépendant sur les deux chaînes.

Les premières mesures ont été effectuées avec une source de ${ }^{241} \mathrm{Am}$ générant un taux de comptage d'environ 20000 coups par seconde, avec un seuil en énergie de $(5,2 \pm 0,5) \mathrm{keV}$ (l'incertitude associée à la position du seuil étant indicative et estimée sur le spectre en énergie). Dans le cas d'un dispositif de déclenchement commun, un écart relatif de 0,025\% est mesuré entre les taux de comptage mesurés sur les deux chaînes. Dans le cas d'un déclenchement indépendant sur chaque chaîne, l'écart relatif mesuré est de 0,06\%. Cet écart plus important traduit les différences de caractéristiques spectrométriques des deux chaînes, et plus particulièrement la définition des seuils.

Afin de vérifier l'efficacité de la gestion des temps morts, la linéarité du taux de comptage en fonction de l'activité a également été étudiée. Pour cela, on effectue plusieurs mesures utilisant des sources étalonnées de ${ }^{137}$ Cs. Pour chaque mesure, l'activité est fixée par le nombre de sources placées dans le détecteur, chaque source contribuant au taux de comptage à hauteur de 10 à 20 milliers de coups par seconde. On utilise un déclenchement indépendant sur chaque chaîne et le seuil est

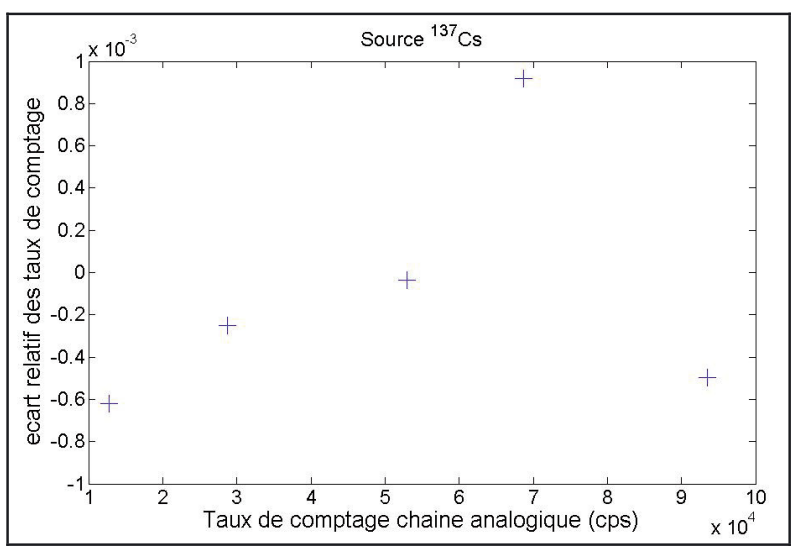

Fig. 3. - Écart relatif entre les taux de comptage mesurés sur la chaîne analogique et la chaîne numérique équipant le cristalpuits en fonction du taux de comptage sur la chaîne analogique.

fixé au-dessus du pic à $32 \mathrm{keV}$ (correspondant aux émissions $\mathrm{X}$ à $31,8 \mathrm{keV}$ et $32,2 \mathrm{keV}$ ) afin de minimiser l'incertitude due à la position des seuils. La figure 3 présente l'écart relatif entre les taux de comptage mesurés sur la chaîne numérique et sur la chaîne analogique en fonction du taux de comptage sur la chaîne analogique déjà validée. On observe un écart relatif inférieur à $0,1 \%$ sur toute la gamme de taux de comptage testée ( de 10 à 100 milliers de coups par seconde).

\subsection{Exemple d'application : instrumentation d'un système RCTD}

Cet exemple s'inscrit dans le développement d'une plate-forme numérique pour la mesure en coïncidences $4 \pi \beta-\gamma$ [12]. La première étape de ce développement concerne l'instrumentation d'une voie «bêta » utilisant les coïncidences obtenues avec un compteur à scintillation liquide à trois photomultiplicateurs à partir duquel il est aussi possible d'appliquer la méthode RCTD. La spécificité des signaux à traiter est principalement leur rapidité, avec une dynamique temporelle de l'ordre de quelques nanosecondes. Dans un premier temps, le dispositif repose sur la mise en forme des signaux par un module analogique de discrimination rapide; les signaux logiques délivrés par ce module peuvent directement alimenter le circuit FPGA. Le comptage des coïncidences entre les photomultiplicateurs peut ainsi être effectué dans le composant programmable en même temps que le traitement des temps morts - de type reconductible avec mesure du temps actif - et la formation de l'histogramme des intervalles de temps actif. On voit que deux éléments diffèrent de l'instrumentation cristal-puits présentée dans la section précédente : la numérisation est effectuée en aval de la discrimination et la totalité des fonctions est exécutée en temps réel.

Le système est constitué d'une carte de développement Altera, équipée d'un FPGA Stratix III, de différents types de mémoires embarquées et de deux connecteurs HSMC (High Speed Mezzanine Connector) permettant l'ajout de modules supplémentaires sur la carte 


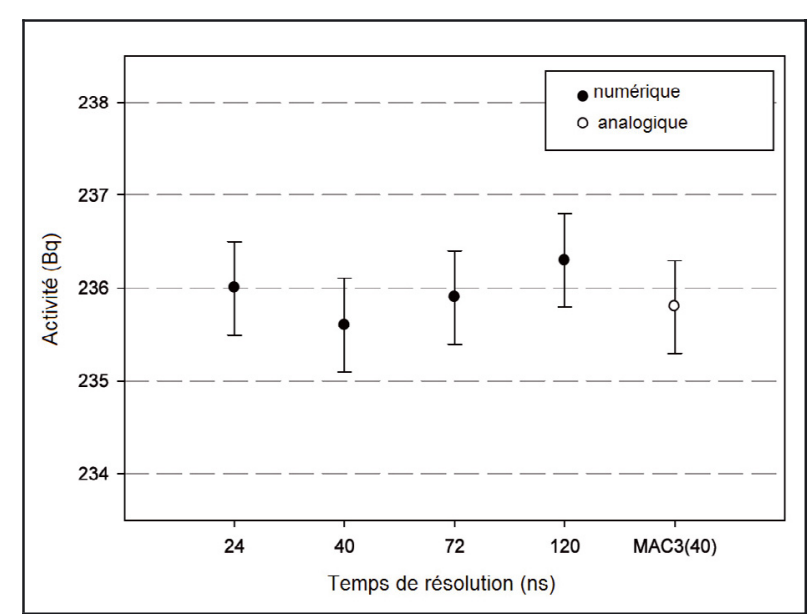

Fig. 4. - Activité d'une source de ${ }^{241}$ Am mesurée par la méthode RCTD sur les chaines analogique (temps de résolution de $40 \mathrm{~ns}$ avec le module MAC3) et numérique (temps de résolution réglable). Les incertitudes correspondent à la statistique de comptage).

principale. Le premier connecteur est utilisé pour la voie dédiée au compteur à scintillation liquide. Le second connecteur est équipé d'un module de numérisation (150 MHz, 14 bits) et d'un module électronique d'entrée élaboré au LNE-LNHB en prévision de l'implémentation prochaine d'une voie gamma.

Ce système a été testé et validé par des mesures en scintillation liquide avec la méthode RCTD sur des sources de ${ }^{241} \mathrm{Am}$ et de ${ }^{3} \mathrm{H}$. Ces mesures ont permis de valider le fonctionnement de l'instrumentation numérique développée, mais également d'explorer les nouvelles fonctionnalités offertes. On rappelle que le temps de résolution de coïncidences est l'intervalle de temps maximal séparant des signaux venant de voies différentes permettant de les accepter comme coïncidents. On présentera ici des résultats concernant l'influence de ce paramètre sur le taux de coïncidences détectées entre les trois photomultiplicateurs du compteur à scintillation liquide. Dans le système analogique, ce temps de résolution est fixé à 40 ns au niveau du module électronique MAC3. Dans le système numérique, sa valeur est modifiable puisqu'elle est fournie par l'utilisateur aux fonctions chargées de la détection des coïncidences dans le FPGA.

La figure 4 présente les résultats de mesures d'activité d'une source de ${ }^{241} \mathrm{Am}$, sur le système numérique pour différents temps de résolution, et sur le système analogique. Les résultats de mesures effectuées sur les deux chaînes sont compatibles, et ce, quel que soit le temps de résolution choisi. L'instrumentation numérique présente une incertitude de mesure comparable à celle de l'instrumentation analogique.

Sur la figure 5 sont présentés des résultats de mesures d'activité pour une source de ${ }^{3} \mathrm{H}$. On constate dans ce cas une nette influence du temps de résolution sur l'activité mesurée. Si le temps de résolution du système numérique est réglé à la valeur utilisée par le système analogique (40 ns), on retrouve bien des résultats compatibles. Cette

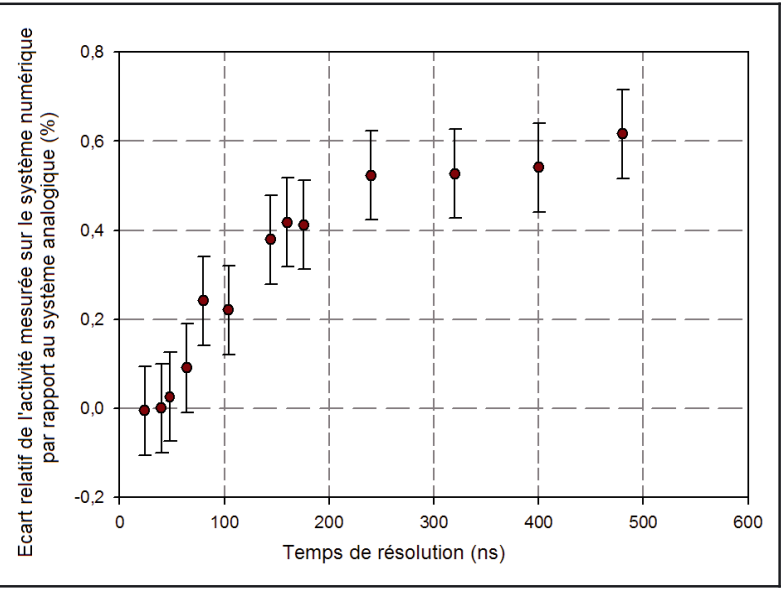

Fig. 5. - Écart relatif des résultats de mesures d'activité d'une source de ${ }^{3} \mathrm{H}$ avec la chaîne numérique par rapport à ceux obtenus avec la chaîne analogique, en fonction du temps de résolution de coïncidences. Les incertitudes correspondent à la statistique de comptage.

influence du temps de résolution est déjà présente dans la mesure du taux de comptage des coïncidences doubles; on est donc en présence d'une variation du taux de comptage qui n'est pas compensée par le modèle RCTD pour le calcul du rendement de détection. Il ne semble pas que les coïncidences fortuites suffisent à rendre compte de l'augmentation du taux de coïncidences en fonction du temps de résolution. De plus, cet effet est lié au nombre moyen de photons émis dans le liquide scintillateur par désintégration du radionucléide mesuré, puisqu'il n'est pas observé pour ${ }^{241} \mathrm{Am}$, dont la désintégration $\alpha$ produit un nombre élevé de photons, ou pour les radionucléides bêta de plus forte énergie. Dans le cas de ${ }^{3} \mathrm{H}$, le nombre de photons émis est faible en raison des basses énergies du rayonnement bêta (énergie maximale de 18,6 keV). Dans ces conditions, le comptage du taux de coïncidences entre les photomultiplicateurs pourrait être sensible à la durée du temps de résolution lorsqu'il n'est pas adapté à la durée de vie des processus de fluorescence dans le scintillateur liquide. On notera que l'existence d'un biais a déjà été rapportée par plusieurs auteurs dans le cas particulier de radionucléides produisant un faible nombre moyen de photons en mesure par scintillation liquide [13], sans qu'il n'ait été apporté d'explication concluante sur son origine. Une étude est en cours au LNE-LNHB pour élucider ce point.

\section{Conclusion}

Les technologies de numérisation rapide et de traitement en temps réel, parvenues à maturité et permettant des applications à l'instrumentation numérique, offrent de nombreuses possibilités pour la mesure d'activité. Concevoir ce type d'instrumentation nécessite cependant une connaissance préalable des méthodes développées et éprouvées jusqu'ici pour les chaînes traditionnelles analogiques. On insistera particulièrement sur les points cruciaux que sont le dispositif électronique d'entrée et le 
traitement des temps morts, qui présentent des contraintes spécifiques au domaine de la métrologie de la radioactivité. Plusieurs travaux ont maintenant établi la faisabilité de telles instrumentations, et annoncent déjà les fonctionnalités supplémentaires qui peuvent être apportées par le numérique.

\section{Références}

[1] KNOLL G.F., "Radiation detection and measurement", John Wiley $\mathcal{F}$ sons, $2^{\mathrm{e}}$ édition, 1989, 120-128.

[2] "Particle counting in radioactivity measurements", International Commission on Radiation Units and measurements, ICRU report 52, novembre 1994.

[3] BOUCHARD J., "MTR2 : a discriminator and dead-time module used in counting systems", Appl. Radiat. and Isot., 52, 2000, 441-446.

[4] Keightley J. et Soon Park T., "Digital coincidence counting for radionuclide standardization", Metrologia, 44, 2007, S32-S35.

[5] Buckman S.M., Keightley J.D., Smith D. et Woods M.J., "The validation of a digital coincidence counting system”, Appl. Radiat. Isot., 49, 1998, 1135-1140.

[6] Hwang H.Y., Park T.S., Kim K.W., JeON W.J., OH P.J., LEE M.K., HAN K.H. et YUN H.J., "An improved method of correlation counting using a bi-dimensional data acquisition system", Nucl. Instrum. Methods A, 369, 1996, 363367.

[7] Keightley J.D. et WatT G.C., "Digital coincidence counting (DCC) and its use in the corrections for outof-channel gamma events in $4 \pi \beta \gamma$ coincidence counting", Appl. Radiat. Isot., 56, 2002, 205-210.

[8] HavelKa M., Auerbach P. et Sochorova J., "Software coincidence counting", Appl. Radiat. Isot., 56, 2002, 265268.

[9] ChernisheV V.I., Korostin S.V., MARTYNYUK YU N., Trofimov A.S. et IORDANSKII P.A., "A digital coincidence method", Meas. Techniq., 47, 2004, 1204-1210.

[10] Havelka M., Auerbach P. et Sochorova J., "Application of pulse mixing method in software coincidence counting", Appl. Radiat. Isot., 60, 2004, 409-413.

[11] Censier B., Bobin C. et Bouchard J., "Digital instrumentation and management of dead time : first results on a NaI-well setup", Appl. Radiat. Isot., 68, 2010, 1314-1319.

[12] Bobin C., BOUChaRd J. et CENSIER B., "First results in the development of an on-line digital counting platform dedicated to primary measurements", Appl. Radiat. Isot., 68, 2010, 1519-1522.

[13] Broda R., Cassette P. et Kossert K., "Radionuclide metrology using liquid scintillation counting", Metrologia, 44, 2007, S36-S52.

Article reçu le 27 juillet 2010, version révisée reçue le 22 décembre 2010. 